\title{
STABILITY FOR RANDOMLY WEIGHTED SUMS OF RANDOM ELEMENTS
}

\author{
TIEN-CHUNG HU and HEN-CHAO CHANG
}

(Received 12 May 1998)

\begin{abstract}
Let $\left\{X_{n}: n=1,2,3, \ldots\right\}$ be a sequence of i.i.d. random elements taking values in a separable Banach space of type $p$ and let $\left\{A_{n, i}: i=1,2,3, \ldots ; n=1,2,3, \ldots\right\}$ be an array of random variables. In this paper, under various assumptions of $\left\{A_{n, i}\right\}$, the necessary and sufficient conditions for $\sum_{i=1}^{\infty} A_{n, i} X_{i} \rightarrow 0$ a.s. are obtained. Also, the necessity of the assumptions of $\left\{A_{n, i}\right\}$ is discussed.
\end{abstract}

Keywords and phrases. Randomly weighted sums, arrays of random elements, almost sure convergence, Banach spaces of type $p$, law of large numbers.

1991 Mathematics Subject Classification. 60B12.

1. Introduction. Let $\left\{X_{n}: n=1,2,3, \ldots\right\}$ be a sequence of independent identically distributed (i.i.d.) random variables and let $\left\{a_{n, i}: i=1,2, \ldots, n ; n=1,2,3, \ldots\right\}$ be a triangular array of constants. Many papers were devoted to extending various types of convergence modes to weighted sums $W_{n}=\sum_{i=1}^{n} a_{n, i} X_{i}$ in the literature. However, we are only interested in the work of almost sure convergence. The sequence $\left\{\sum_{i=1}^{n} a_{n, i}\right\}$ converging to 0 at a certain rate as $n \rightarrow \infty$ is a traditional assumption. For example, under the assumption $\sum_{i=1}^{n} a_{n, i}^{2}=O\left(n^{-2 / r}\right), W_{n} \rightarrow 0$ a.s. if $E\left|X_{1}\right|^{r}<\infty$ and $E X_{1}=0$ (See Chow and Lia [3] and Choi and Sung [2]). On the other hand, Padgett and Taylor [4] extended the usual convergence theorems to weighted sums of random elements in a separable Banach space. It would be interesting to extend the results with random weights.

Let $\left\{A_{n, i}: i=1,2, \ldots, n ; n=1,2,3, \ldots\right\}$ be a triangular array of random variables such that $\sum_{i=1}^{n} A_{n, i}^{2}=O\left(n^{-2 / r}\right)$ a.s., Ahmad [1] obtained $W_{n}=\sum_{i=1}^{n} A_{n, i} X_{i} \rightarrow 0$ a.s. if $E\left\|X_{1}\right\|^{r}<\infty$ and $E X_{1}=0$. We note that, for the Marcinkiewicz-Zygmund law of large numbers, we take the uniform weight $a_{n, i}=n^{-1 / r}$ but the condition $\sum_{i=1}^{n} a_{n, i}^{2}=$ $O\left(n^{-2 / r}\right)$ cannot be satisfied. The purpose of this paper is to extend the randomly weighted sums of a triangular array of random variables to that of an infinite array of random elements such that the Marcinkiewicz-Zygmund law of large numbers can be obtained as a corollary.

In Section 2, we establish the Marcinkiewicz-Zygmund law of large numbers in a separable Banach space of Type $p$. In Section 3, we consider an infinite array of random variables $\left\{A_{n, i}: n, i=1,2,3, \ldots\right\}$ as the weight under various assumptions of $\left\{A_{n, i}\right\}$, we obtain that $W_{n}=\sum_{i=1}^{\infty} A_{n, i} X_{i} \rightarrow 0$ a.s. if and only if $E X_{1}=0$ (when it exists) and $E\left\|X_{1}\right\|^{r}<\infty$. 
2. The Marcinkiewicz law in a space of type $p$. Let $(\Omega, F, P)$ be a probability space and $\mathbf{B}$ be a real separable Banach space with norm $\|\bullet\|$. A random element is defined to be an $F$-measurable mapping of $\Omega$ into B with the Borel $\sigma$-field. The concept of independent random elements is a direct extension of the concept of independent random variables. A detailed account of basic properties of random elements in real Banach spaces can be found in Taylor [6].

In this section, we prove the Marcinkiewicz-Zygmund law of large numbers in a space of type $p$. First, we introduce a space of type $p$.

DEFINITION 1. Let $1 \leq p \leq 2$ and $\left\{r_{i}: i=1,2,3, \ldots\right\}$ be a sequence of independent random variables with $\operatorname{Pr}\left(r_{i}= \pm 1\right)=1 / 2$. A separable Banach space $\mathbf{B}$ is said to be of type $p$ if there exists a constant $C$ such that

$$
E\left\|\sum_{i=1}^{n} r_{i} x_{i}\right\| \leq C\left(\sum_{i=1}^{n}\left\|x_{i}\right\|^{p}\right)^{1 / p}
$$

for every $n \in N$ and all $x_{1}, \ldots, x_{n} \in \mathbf{B}$.

Woyczyński [7] proved the equivalent condition of a space of type $p$.

LEMMA 1 (Woyczyński [7]). Let $1 \leq p \leq 2$ and $q \geq 1$. The following properties of $\mathbf{B}$ are equivalent :

(i) The separable Banach space $\mathrm{B}$ is of type $p$.

(ii) There exists $C$ such that, for every $n \in N$ and for any sequence $\left\{X_{i}: i=1,2, \ldots, n\right\}$ of independent random elements in $\mathbf{B}$ with $E X_{i}=0, i=1,2, \ldots, n$,

$$
E\left(\left\|\sum_{i=1}^{n} X_{i}\right\|^{q}\right) \leq C E\left(\left(\sum_{i=1}^{n}\left\|X_{i}\right\|^{p}\right)^{q / p}\right) .
$$

Using Lemma 1, some elementary properties of spaces of type $p$ can be easily proved. Every separable Hilbert space and finite-dimensional Banach space are of type 2. Every separable Banach space is at least of type 1 , and the $\ell^{p}$ and $L^{p}$ are of type $\min \{2, p\}$ for $p \geq 1$. If $\mathbf{B}$ is a space of type $p$ and $1 \leq q \leq p$, then $\mathbf{B}$ is a space of type $q$. Before considering the Marcinkiewicz-Zygmund law of large numbers in a space of type $p$, we need the following definition and lemmas.

Definition 2. Let $\mathbf{B}$ be a separable Banach space, $\mathbf{B}^{*}$ the dual space of $\mathbf{B}$, and $\mathbf{B}^{\prime}$ the unit ball in $\mathbf{B}^{*} . X$ is a random element in $\mathbf{B}$. The directionally maximum median of $X$ is defined by

$$
\rho(X) \equiv \sup _{f \in \mathbf{B}^{\prime}}|\mu(f(X))|,
$$

where $\mu(Y)$ denotes the minimum median in absolute value of the random variable $Y$.

LEMMA 2 (Sakhanenko [5]). Let $X_{1}, \ldots, X_{n}$ be independent random elements in $\mathbf{B}$ and $S_{k}=\sum_{i=1}^{k} X_{i}$, then, for every $t>0$,

$$
\operatorname{Pr}\left(\max _{1 \leq k \leq n}\left\|S_{k}\right\|>t\right) \leq 2 \operatorname{Pr}\left(\left\|S_{n}\right\|>t-\max _{1 \leq k \leq n} \rho\left(S_{n}-S_{k}\right)\right) .
$$


LEMмA 3. Let $\left\{X_{n}: n=1,2,3, \ldots\right\}$ be a sequence of independent random elements in a separable Banach space. If $S_{n}=\sum_{i=1}^{n} X_{i}$ converges to a random element $S$ in probability, then $S_{n}$ converges to $S$ a.s.

Proof. Since $S_{n}$ converges to $S$ in probability, take $\epsilon$ such that $0<\epsilon<1 / 2$, then there exists an integer $n_{0}$ such that if $m>n \geq n_{0}$,

$$
\operatorname{Pr}\left(\left\|S_{m}-S\right\|>\frac{\epsilon}{2}\right)<\frac{\epsilon}{2} \quad \text { and } \quad \operatorname{Pr}\left(\left\|S_{n}-S\right\|>\frac{\epsilon}{2}\right)<\frac{\epsilon}{2} .
$$

So,

$$
\operatorname{Pr}\left(\left\|S_{m}-S_{n}\right\|>\epsilon\right)<\operatorname{Pr}\left(\left\|S_{m}-S\right\|>\frac{\epsilon}{2}\right)+\operatorname{Pr}\left(\left\|S_{n}-S\right\|>\frac{\epsilon}{2}\right)<\epsilon<\frac{1}{2} .
$$

We have $\mu\left(\left\|S_{m}-S_{n}\right\|\right)<\epsilon$ for any $m>n \leq n_{0}$, where $\mu(Y)$ is minimum median in absolute value of the random variable $Y$.

By Lemma 2, if $m_{1}>n>n_{0}$,

$$
\begin{aligned}
\operatorname{Pr}\left(\max _{n<m<m_{1}}\left\|S_{m}-S_{n}\right\|>2 \epsilon\right) & \leq 2 \operatorname{Pr}\left(\left\|S_{m_{1}}-S_{n}\right\|>2 \epsilon-\max _{n<m<m_{1}} \rho\left(S_{m_{1}}-S_{m}\right)\right) \\
& \leq 2 \operatorname{Pr}\left(\left\|S_{m_{1}}-S_{n}\right\|>2 \epsilon-\max _{n<m<m_{1}} \mu\left(\left\|S_{m_{1}}-S_{m}\right\|\right)\right) \\
& \leq 2 \operatorname{Pr}\left(\left\|S_{m_{1}}-S_{n}\right\|>\epsilon\right)<2 \epsilon .
\end{aligned}
$$

Let $m_{1} \rightarrow \infty$, then if $m>n>n_{0}$, we have $\operatorname{Pr}\left(\max _{n<m}\left\|S_{m}-S_{n}\right\|>2 \epsilon\right)<2 \epsilon$.

We obtain $S_{n}$ converges to some random element a.s., and $S_{n}$ converges to $S$ in probability. Hence, $S_{n}$ converges to $S$ a.s.

Now, we prove the Marcinkiewicz-Zygmund law of large numbers in a space of type $p$.

THEOREM 1. Let B be a separable Banach space of type $P$ and $\left\{X_{n}: n=1,2,3, \ldots\right\}$ be a sequence of independent and identically distributed random elements in $\mathbf{B}$. Then, for any $0<r<p$,

$$
\frac{S_{n}-n c}{n^{1 / r}} \longrightarrow 0 \text { a.s. }
$$

for some constant $c$ if and only if $E\left\|X_{1}\right\|^{r}<\infty$.

Moreover, if $r \geq 1, c=E X_{1}$ and $0<r<1, c$ is arbitrary.

\section{ProOF.}

NECESSARY PART. Since (2.8) holds,

$$
\frac{X_{n}}{n^{1 / r}}=\frac{S_{n}-n c}{n^{1 / r}}-\left(\frac{n-1}{n}\right)^{1 / r} \frac{S_{n-1}-n c}{(n-1)^{1 / r}} \rightarrow 0 \quad \text { a.s., }
$$

whence, by the Borel-Cantelli lemma, $\sum_{n=1}^{\infty} \operatorname{Pr}\left(\left\|X_{1}\right\|>n^{1 / r}\right)<\infty$. Thus, $E\left\|X_{1}\right\|^{r}<\infty$.

SUFFICIENT PART. Since $E\left\|X_{1}\right\|^{r}<\infty$, define $Y_{n}=n^{-1 / r} X_{n} I\left(\left\|X_{n}\right\| \leq n^{1 / r}\right)$ and $A_{j}=$ $\left\{(j-1)^{1 / r}<\left\|X_{1}\right\| \leq j^{1 / r}\right\}$. Choose a positive number $\alpha$ such that $r<\alpha \leq p$ and $\alpha \geq 1$. We have

$$
\sum_{n=1}^{\infty} E\left\|Y_{n}\right\|^{\alpha}=\sum_{n=1}^{\infty} \sum_{j=1}^{n} n^{-\alpha / r} \int_{A_{j}}\left\|X_{1}\right\|^{\alpha} d p=\sum_{j=1}^{\infty} \sum_{n=j}^{\infty} n^{-\alpha / r} \int_{A_{j}}\left\|X_{1}\right\|^{\alpha} d p
$$




$$
\begin{aligned}
& \leq c_{1} \sum_{j=1}^{\infty} j^{1-\alpha / r} \int_{A_{j}}\left\|X_{1}\right\|^{\alpha} d p \leq c_{1} \sum_{j=1}^{\infty} \int_{A_{j}}\left\|X_{1}\right\|^{r} d p \\
& =c_{1} E\left\|X_{1}\right\|^{r}<\infty, \quad \text { for some constant } c_{1} .
\end{aligned}
$$

From Lemma 1,

$$
\begin{aligned}
E\left\|\sum_{i=n}^{m}\left(Y_{i}-E Y_{i}\right)\right\|^{\alpha} & \leq c_{2} E\left(\sum_{i=n}^{m}\left\|Y_{i}-E Y_{i}\right\|^{p}\right)^{\alpha / p} \\
& \leq c_{2} \sum_{i=n}^{m} E\left\|Y_{i}-E Y_{i}\right\|^{\alpha} \leq 2^{\alpha} c_{2} \sum_{i=n}^{m} E\left\|Y_{i}\right\|^{\alpha} .
\end{aligned}
$$

We have $\sum_{i=1}^{n}\left(Y_{i}-E Y_{i}\right)$ converges to some random element $Y_{0}$ in $L^{\alpha}$. Therefore, $\sum_{i=1}^{n}\left(Y_{i}-E Y_{i}\right) \rightarrow Y_{0}$ in probability. By Lemma 3, $\sum_{i=1}^{n}\left(Y_{i}-E Y_{i}\right) \rightarrow Y_{0}$ a.s. Since

$$
\sum_{n=1}^{\infty} \operatorname{Pr}\left(\frac{X_{n}}{n^{1 / r}} \neq Y_{n}\right)=\sum_{n=1}^{\infty} \operatorname{Pr}\left(\left\|X_{1}\right\|>n^{1 / r}\right) \leq E\left\|X_{1}\right\|^{r}<\infty,
$$

so,

$$
\sum_{n=1}^{\infty} \frac{X_{n}-E\left(X_{n} I\left(\left\|X_{n}\right\| \leq n^{1 / r}\right)\right)}{n^{1 / r}}=\sum_{n=1}^{\infty}\left(\frac{X_{n}}{n^{1 / r}}-E Y_{n}\right) \quad \text { converges a.s. }
$$

If $0<r<1$, we can choose $\alpha=1$. Then $\sum_{n=1}^{\infty} E\left\|Y_{n}\right\|<\infty$. We have $\sum_{n=1}^{\infty}\left(X_{n}\right) /\left(n^{1 / r}\right)$ converges a.s. By Kronecker lemma, $\left(S_{n}-n c\right) /\left(n^{1 / r}\right)$ converges a.s. for any constant $c$.

If $r=1$, by Kronecker lemma, $\left(S_{n} / n\right)-(1 / n) \sum_{i=1}^{n} E\left(X_{i} I\left(\left\|X_{i}\right\| \leq n\right)\right)$ converges a.s. and $E\left(X_{n} I\left(\left\|X_{n}\right\| \leq n\right)\right) \rightarrow E X_{1}$, we have (2.8) holds.

If $r>1$, we can show that

$$
\begin{array}{rl}
\sum_{n=1}^{\infty} n^{-1 / r} & E\left\|X_{n} I\left(\left\|X_{n}\right\|>n^{1 / r}\right)\right\| \\
& \leq \sum_{n=1}^{\infty} n^{-1 / r} E\left(\left\|X_{1}\right\| I\left(\left\|X_{1}\right\|>n^{1 / r}\right)\right)=\sum_{n=1}^{\infty} n^{-1 / r} \sum_{j=n+1}^{\infty} \int_{A_{j}}\left\|X_{1}\right\| d p \\
& =\sum_{j=2}^{\infty} \sum_{n=1}^{j-1} n^{-1 / r} \int_{A_{j}}\left\|X_{1}\right\| d p \leq \frac{r}{r-1} \sum_{j=1}^{\infty}(j-1)^{(r-1) / r} \int_{A_{j}}\left\|X_{1}\right\| d p \\
& \leq \frac{r}{r-1} \sum_{j=1}^{\infty} \int_{A_{j}}\left\|X_{1}\right\|^{r} d p=\frac{r}{r-1} E\left\|X_{1}\right\|^{r}<\infty
\end{array}
$$

Therefore, $\sum_{n=1}^{\infty}\left(X_{n}-E X_{1}\right) / n^{1 / r}$ converges a.s. We have (2.8) holds by Kronecker lemma.

3. The convergence of the weighted sums. Throughout this section, we deal with the almost sure convergence of randomly weighted sums $\sum_{i=1}^{\infty} A_{n, i} X_{i}$, where $\left\{X_{n}\right.$ : $n=1,2,3, \ldots\}$ is a sequence of independent and identically distributed random elements in a space of type $p$ and $\left\{A_{n, i}: n, i=1,2,3, \ldots\right\}$ is an array of random variables satisfying some conditions. 
THEOREM 2. Let B be a separable Banach space of type $p$. Let $\left\{X_{n}: n=1,2,3, \ldots\right\}$ be a sequence of independent and identically distributed random elements in $\mathbf{B}$ such that $E\left\|X_{1}\right\|^{r}<\infty$ and $r<p$. Moreover, we assume that $E X_{1}=0$ when $r \geq 1$. Let $\left\{A_{n, i}: n, i=\right.$ $1,2,3, \ldots\}$ be an array of random variables such that $\left\{A_{n, i}\right\}$ and $\left\{X_{i}\right\}$ are independent and satisfying

and

$$
\begin{array}{cc}
A_{n, i}=O\left(i^{-1 / r}\right) & \text { a.s. for every } n, \\
\lim _{n \rightarrow \infty} A_{n, i}=0 \quad \text { a.s. for every } i, \\
\sum_{i=1}^{\infty} E\left|A_{n, i}\right|^{r}<\infty & \text { for every } n,
\end{array}
$$

$$
\sum_{i=1}^{\infty} i^{1 / r}\left|A_{n, i}-A_{n, i+1}\right|<M \quad \text { a.s. for every } n \text { and some constant } M>0 .
$$

Then

Conversely, if

$$
\lim _{n \rightarrow \infty} \sum_{i=1}^{\infty} A_{n, i} X_{i}=0 \quad \text { a.s. }
$$

$$
\lim _{n \rightarrow \infty} \sum_{i=1}^{\infty} A_{n, i} X_{i}=0 \quad \text { a.s. }
$$

for all arrays $\left\{A_{n, i}\right\}$ satisfying the above conditions, then $E\left\|X_{1}\right\|^{r}<\infty$.

Proof. Since $\left\{A_{n, i}\right\}$ and $\left\{X_{i}\right\}$ are independent, if $r>1$, we choose $p=q=r$ in Lemma 1, then

$$
E\left(\sum_{i=1}^{\infty}\left\|A_{n, i} X_{i}\right\|\right)^{r} \leq \sum_{i=1}^{\infty} E\left|A_{n, i}\right|^{r} E\left\|X_{1}\right\|^{r}<\infty .
$$

If $r \leq 1$, it is obvious that

$$
E\left(\sum_{i=1}^{\infty}\left\|A_{n, i} X_{i}\right\|\right)^{r} \leq \sum_{i=1}^{\infty} E\left|A_{n, i}\right|^{r} E\left\|X_{1}\right\|^{r}<\infty .
$$

Therefore, $\sum_{i=1}^{\infty} A_{n, i} X_{i}$ converges a.s.

Since $A_{n, i}=A_{n, i}^{+}-A_{n, i}^{-}$, without loss of generality, we can assume that $A_{n, i} \geq 0$. Let $S_{k}=\sum_{i=1}^{k} X_{i}, Y_{k}=\left(S_{k} / k^{1 / r}\right)$ for every $k \geq 1$ and $S_{0}=0$. By Theorem 1 , we have $\lim _{k \rightarrow \infty} Y_{k}=0$ a.s.

$$
\sum_{i=1}^{\infty} A_{n, i} X_{i}=\sum_{i=1}^{\infty} A_{n, i}\left(S_{i}-S_{i-1}\right)=\lim _{N \rightarrow \infty}\left(\sum_{i-1}^{N-1}\left(A_{n, i}-A_{n, i+1}\right) S_{i}+A_{n, N} S_{N}\right) .
$$

Since $\left\{i^{1 / r} A_{n, i}\right\}$ is bounded a.s. for every $n$ and $r$,

$$
\lim _{N \rightarrow \infty} A_{n, N} S_{N}=\lim _{N \rightarrow \infty}\left(N^{1 / r} A_{n, N}\right) Y_{N}=0 \text { a.s. }
$$

We have

$$
\sum_{i=1}^{\infty} A_{n, i} X_{i}=\sum_{i=1}^{\infty} i^{1 / r}\left(A_{n, i}-A_{n, i+1}\right) Y_{i} \text { a.s. }
$$


Let $B_{n, i}=i^{1 / r}\left(A_{n, i}-A_{n, i+1}\right)$. Hence, $\sum_{i=1}^{\infty}\left|B_{n, i}\right| \leq M$ a.s. for every $n$ and $\lim _{n \rightarrow \infty} B_{n, i}=0$ a.s. for every $i$. Define $D^{0}=\left\{w: \lim _{i \rightarrow \infty} Y_{i}(w)=0\right\}$ and $D_{n}^{1}=\left\{w: \sum_{i=1}^{\infty}\left|B_{n, i}(w)\right| \leq M\right\}$ for each $n$ and $D_{i}^{2}=\left\{w: \lim _{n \rightarrow \infty} B_{n, i}(w)=0\right\}$ for each $i$. For every $w \in D^{0} \cap \bigcap_{i=1}^{\infty}\left(D_{i}^{1} \cap\right.$ $D_{i}^{2}$ ) and every $\epsilon>0$, we can choose $A$ such that $\left\|Y_{i}(w)\right\|<\epsilon$ for $i \geq A$,

$$
\begin{aligned}
\sum_{i=1}^{\infty}\left\|B_{n, i}(w) Y_{i}(w)\right\| & \leq \sum_{i=1}^{A-1}\left|B_{n, i}(w)\right|\left\|Y_{i}(w)\right\|+\sum_{i=A}^{\infty}\left|B_{n, i}(w)\right|\left\|Y_{i}(w)\right\| \\
& \leq \max _{i \leq A-1}\left\|Y_{i}(w)\right\| \sum_{i=1}^{A-1}\left|B_{n, i}(w)\right|+M \epsilon \rightarrow M \epsilon \quad \text { as } n \longrightarrow \infty
\end{aligned}
$$

Since $\operatorname{Pr}\left(D^{0} \cap \bigcap_{i=1}^{\infty}\left(D_{i}^{1} \cap D_{i}^{2}\right)\right)=1, \lim _{n \rightarrow \infty} \sum_{i=1}^{\infty}\left\|B_{n, i} Y_{i}\right\|=0$ a.s. Therefore,

$$
\lim _{n \rightarrow \infty} \sum_{i=1}^{\infty} A_{n, i} X_{i}=\lim _{n \rightarrow \infty} \sum_{i=1}^{\infty} B_{n, i} Y_{i}=0 \text { a.s. }
$$

If $\lim _{n \rightarrow \infty} \sum_{i=1}^{\infty} A_{n, i} X_{i}=0$ a.s. for all arrays $\left\{A_{n, i}\right\}$ satisfying the above conditions, we can choose

$$
A_{n, i}= \begin{cases}n^{-1 / r} & \text { if } i \leq n, \\ 0 & \text { if } i>n\end{cases}
$$

Then (2.8) holds. By Theorem 1, we have $E\left\|X_{1}\right\|^{r}<\infty$.

REMARK 1. The following example claims that condition (3.4) cannot be omitted. Consider the real number space $R$ as a space of type 2 . Choose a sequence $\left\{X_{n}: n=\right.$ $1,2,3, \ldots\}$ of independent and identically distributed random variables with $E X_{1}^{2}<\infty$ and $E X_{1}=0$. Define

$$
A_{n, i}= \begin{cases}n^{-1 / 2} & \text { if } i \leq n, \\ 0 & \text { if } i>n .\end{cases}
$$

Choose any $r<2$ so that condition (3.4) does not hold. By the Central Limit Theorem, $\sum_{i=1}^{\infty} A_{n, i} X_{i}$ cannot converge to 0 a.s.

Choi and Sung [2] considered the almost sure convergence of $\sum_{i=1}^{\infty} a_{n, i} X_{i}$ for triangular array of constants. Their Theorem 3 can be regarded as a corollary of Theorem 2.

Corollary 1 (Choi and Sung [2, Theorem 3]). Let $\left\{X_{n}: n=1,2,3, \ldots\right\}$ be independent and identically distributed random variables with $E X_{1}=0$ and $E\left|X_{1}\right|^{r}<\infty$ for some $1 \leq r<2$. Let $\left\{a_{n, i}: i=1,2, \ldots, n ; n=1,2,3, \ldots\right\}$ be a triangular array of constants satisfying $\sum_{i=1}^{n}\left|a_{n, i}-a_{n, i+1}\right|=O\left(n^{-1 / r}\right)$, where $a_{n, n+1}=0$. Then

$$
\lim _{n \rightarrow \infty} \sum_{i=1}^{n} a_{n, i} X_{i}=0 \quad \text { a.s. }
$$

Proof. By Theorem 2, we must show that there is a constant $M>0$ such that

$$
\lim _{n \rightarrow \infty} a_{n, i}=0 \quad \text { for every } i
$$


and

$$
\sum_{i=1}^{\infty} i^{1 / r}\left|a_{n, i}-a_{n, i+1}\right|<M \text { for every } n .
$$

There is a constant $C>0$ such that $\sum_{i=1}^{n}\left|a_{n, i}-a_{n, i+1}\right| \leq C n^{-1 / r}$. We have $\left|a_{n, i}\right| \leq$ $C n^{-1 / r}$ for every $i$. So, $\lim _{n \rightarrow \infty} a_{n, i}=0$ for every $i$.

Therefore,

$$
\begin{aligned}
\sum_{i=1}^{\infty} i^{1 / r}\left|a_{n, i}-a_{n, i+1}\right| & =\sum_{i=1}^{n} i^{1 / r}\left|a_{n, i}-a_{n, i+1}\right| \\
& \leq n^{1 / r} \sum_{i=1}^{n}\left|a_{n, i}-a_{n, i+1}\right| \leq C .
\end{aligned}
$$

So, the proof is complete.

The assumptions of $\left\{A_{n, i}\right\}$ in Theorem 2 can be simplified as in Theorem 3 for $r<1$ and Theorem 4 for $r \geq 1$.

LEMMA 4. Let $\left\{b_{n}: n=1,2,3, \ldots\right\}$ be a sequence of positive numbers. If $\sum_{i=1}^{\infty} i \mid b_{i}-$ $b_{i+1} \mid<\infty$ and $\sum_{i=1}^{\infty} b_{i}<\infty$, then there exists $C>0$ such that $i b_{i}<C$ for all $i$.

Proof. Since $\sum_{i=1}^{\infty} i\left|b_{i}-b_{i+1}\right|<\infty$, there exists $N>0$ such that $\sum_{k=N}^{\infty} i\left|b_{i}-b_{i+1}\right|<$ 1. If the result of this Lemma is false, then any $n, l>0$, there exists $i>l$ such that $b_{i}>n / i$. We define

$$
n_{i} \equiv \inf \left\{i: i>2 n_{j-1} \text { and } b_{i}>\frac{J}{i}\right\} \quad \text { if } j \geq 2
$$

And

$$
n_{0} \equiv 0, \quad n_{1} \equiv \inf \left\{i: i>N \text { and } b_{i}>\frac{1}{i}\right\}
$$

We see that

$$
\sum_{i=1}^{\infty} b_{i}=\sum_{i=1}^{\infty} \sum_{k=n_{i-1}+1}^{n_{i}} b_{k}=\sum_{i=1}^{\infty} \sum_{k=n_{i-1}+1}^{n_{i}}\left(b_{n_{i}}+\left(b_{k}-b_{n_{i}}\right)\right) .
$$

If $m>n \geq N$, then

$$
\sum_{i=n}^{m} b_{i}-b_{m} \leq \sum_{i=n}^{m} \sum_{k=i}^{m-1}\left|b_{k}-b_{k+1}\right|=\sum_{k=n}^{m-1} \sum_{i=n}^{k}\left|b_{k}-b_{k+1}\right| \leq \sum_{k=n}^{m-1} K\left|b_{k}-b_{k+1}\right|<1 .
$$

Therefore,

$$
\begin{aligned}
\sum_{i=1}^{\infty} b_{i} & =\sum_{i=1}^{\infty} \sum_{k=n_{i-1}+i}^{n_{i}}\left(b_{n_{i}}+\left(b_{k}-b_{n_{i}}\right)\right) \\
& \geq \sum_{i=2}^{\infty}\left(\frac{i}{n_{i}}\left(n_{i}-n_{i-1}\right)-1\right) \geq \sum_{i=2}^{\infty}\left(\frac{i}{n_{i}} \times \frac{n_{i}}{2}-1\right)=\infty .
\end{aligned}
$$

But $\sum_{i=1}^{\infty} b_{i}<\infty$ and the proof is complete.

When $r<1$, Theorem 2 can be rewritten as follows. 
THEOREM 3. Let B be a separable Banach space of type $p$. Let $\left\{X_{n}: n=1,2,3, \ldots\right\}$ be a sequence of independent and identically distributed random elements in $\mathbf{B}$ such that $E\left\|X_{1}\right\|^{r}<\infty$ and $r<1$. Let $\left\{A_{n, i}: n, i=1,2,3, \ldots\right\}$ be an array of random variables such that $\left\{A_{n, i}\right\}$ and $\left\{X_{i}\right\}$ are independent, and satisfying

$$
\begin{gathered}
\lim _{n \rightarrow \infty} A_{n, i}=0 \quad \text { a.s. for every } i, \\
\sum_{i=1}^{\infty} E\left|A_{n, i}\right|^{r}<\infty \quad \text { a.s. for every } n
\end{gathered}
$$

and

$$
\sum_{i=1}^{\infty} i\left|A_{n, i}-A_{n, i+1}\right|^{r}<M \quad \text { a.s. for every } n \text { and some constant } M>0 .
$$

Then $\lim _{n \rightarrow \infty} \sum_{i=1}^{\infty} A_{n, i} X_{i}=0$ a.s.

Conversely, if $\lim _{n \rightarrow \infty} \sum_{i=1}^{\infty} A_{n, i} X_{i}=0$ a.s. for all arrays $\left\{A_{n, i}\right\}$ satisfying the above conditions, then $E\left\|X_{1}\right\|^{r}<\infty$.

Proof. Since $A_{n, i}=A_{n, i}^{+}-A_{n, i}^{-}$, without loss of generality, we can assume that $A_{n, i} \geq 0$. We consider $A_{n, i}^{r}=b_{i}$ in Lemma 4 . Since $\sum_{i=1}^{\infty} i\left|A_{n, i}^{r}-A_{n, i+1}^{r}\right| \leq \sum_{i=1}^{\infty} i \mid A_{n, i}-$ $\left.A_{n, i+1}\right|^{r}$, for $r<1$, we have $A_{n, i}=O\left(i^{-1 / r}\right)$ a.s. for every $n$. From the proof of Theorem 2, we have $\lim _{i \rightarrow \infty} Y_{i}=0$ a.s. and $\sum_{i=1}^{\infty} A_{n, i} X_{i}=\sum_{i=1}^{\infty} i^{1 / r}\left(A_{n, i}-A_{n, i+1}\right) Y_{i}$ a.s., where $Y_{i}=\left(1 / i^{1 / r}\right) \sum_{j=1}^{i} X_{j}$.

Define $B_{n, i}=i^{1 / r}\left(A_{n, i}-A_{n, i+1}\right)$. Hence, $\sum_{i=1}^{\infty}\left|B_{n, i}\right|^{r} \leq M$ a.s. and $\lim _{n \rightarrow \infty} B_{n, i}=0$ a.s. Let $D=D^{0} \cap \bigcap_{i=1}^{\infty}\left(D_{i}^{1} \cap D_{i}^{2}\right)$, where the definitions of $D^{0}, D_{i}^{1}$, and $D_{i}^{2}$ are the same as in Theorem 2. For every $w \in D$ and every $\epsilon>0$, we can choose $A$ such that $\left\|Y_{i}(w)\right\|<\epsilon$ for $i \geq A$,

$$
\begin{aligned}
\sum_{i=1}^{\infty}\left\|B_{n, i} Y_{i}(w)\right\|^{r} & \leq \sum_{i=1}^{A-1}\left|B_{n, i}\right|^{r}\left\|Y_{i}(w)\right\|^{r}+\sum_{i=A}^{\infty}\left|B_{n, i}\right|^{r}\left\|Y_{i}(w)\right\|^{r} \\
& \leq \max _{i \leq A-1}\left\|Y_{i}(w)\right\|^{r} \sum_{i=1}^{A-1}\left|B_{n, i}\right|^{r}+M \epsilon \longrightarrow M \epsilon \quad \text { as } n \longrightarrow \infty .
\end{aligned}
$$

Since $\operatorname{Pr}(D)=1, \lim _{n \rightarrow \infty} \sum_{i=1}^{\infty}\left\|B_{n, i} Y_{i}\right\|^{r}=0$ a.s. Therefore,

$$
\lim _{n \rightarrow \infty} A_{n, i} X_{i}=\lim _{n \rightarrow \infty} \sum_{i=1}^{\infty} B_{n, i} Y_{i}=0 \quad \text { a.s. }
$$

The proof of the converse part is the same as the proof of Theorem 2. So the proof is complete.

When $r \geq 1$, we can obtain the following theorem:

THEOREM 4. Let B be a separable Banach space of type $p$. Let $\left\{X_{n}: n=1,2,3, \ldots\right\}$ be a sequence of independent and identically distributed random elements in $\mathbf{B}$ such that $E X_{1}=0$ and $E\left\|X_{1}\right\|^{r}<\infty$ for $1 \leq r<p$. Let $\left\{A_{n, i}: n, i=1,2,3, \ldots\right\}$ be an array of 
random variables such that $\left\{A_{n, i}\right\}$ and $\left\{X_{i}\right\}$ are independent, and satisfying

$$
\begin{aligned}
& \lim _{n \rightarrow \infty} A_{n, i}=0 \quad \text { a.s. for every } i, \\
& \sum_{i=1}^{\infty} E\left|A_{n, i}\right|^{r}<\infty \text { for every } n,
\end{aligned}
$$

and

$$
\sum_{i=1}^{\infty} i^{1 / r}\left|A_{n, i}^{r}-A_{n, i+1}^{r}\right|^{1 / r}<M \quad \text { a.s. for every } n \text { and some constant } M>0 .
$$

Then

Conversely, if

$$
\lim _{n \rightarrow \infty} \sum_{i=1}^{\infty} A_{n, i} X_{i}=0 \quad \text { a.s. }
$$

$$
\lim _{n \rightarrow \infty} \sum_{i=1}^{\infty} A_{n, i} X_{i}=0 \quad \text { a.s. }
$$

for all arrays $\left\{A_{n, i}\right\}$ satisfying the above conditions, then $E\left\|X_{1}\right\|^{r}<\infty$.

Proof. We see that

and

$$
\sum_{i=1}^{\infty} i^{1 / r}\left|A_{n, i}-A_{n, i+1}\right|<\sum_{i=1}^{\infty} i^{1 / r}\left|A_{n, i}^{r}-A_{n, i+1}^{r}\right|^{1 / r}<M
$$

$$
\sum_{i=1}^{\infty} i^{1 / r}\left|A_{n, i}^{r}-A_{n, i+1}^{r}\right|^{1 / r}<\infty \Longrightarrow \sum_{i=1}^{\infty} i\left|A_{n, i}^{r}-A_{n, i+1}^{r}\right|<\infty \quad \text { (since } r \geq 1 \text { ). }
$$

So, from the proofs of Theorem 2 and Theorem 3, we can obtain this theorem.

Now, we consider a very special case of $\left\{A_{n, i}\right\}$. Let $A_{n, i}=n^{-1 / r}$ for $i=1,2, \ldots, n$ and $A_{n, i}=0$ for $i>n$. The assumptions of $\left\{A_{n, i}\right\}$ in Theorem 4 can be easily verified. Therefore, the Marcinkiewicz-Zygmund law of large numbers in a space of type $p$ can be obtained as the following corollary.

COROLLARY 2. Let $\mathbf{B}$ be a separable Banach space of type $p$ and $\left\{X_{n}: n=1,2,3, \ldots\right\}$ be a sequence of independent and identically distributed random elements in $\mathbf{B}$ with zero means. For any $1 \leq r<p$, we have if $E\left\|X_{1}\right\|^{r}<\infty$, then

$$
\left(\frac{1}{n}\right)^{1 / r} \sum_{i=1}^{n} X_{i} \rightarrow 0 \text { a.s. }
$$

Proof. Let $A_{n, i}=n^{-1 / r}$ for $i=1,2, \ldots, n$ and $A_{n, i}=0$ for $i>n$. Since

and

$$
\begin{gathered}
\lim _{n \rightarrow \infty} A_{n, i}=\lim _{n \rightarrow \infty} n^{-1 / r}=0, \\
\sum_{i=1}^{\infty} E\left|A_{n, i}\right|^{r}=\sum_{i=1}^{n} \frac{1}{n}=1,
\end{gathered}
$$

$$
\sum_{i=1}^{\infty} i^{1 / r}\left|A_{n, i}^{r}-A_{n, i+1}^{r}\right|^{1 / r}=n^{1 / r} \cdot n^{-1 / r}=1,
$$

the proof is complete by Theorem 4 . 


\section{REFERENCES}

[1] I. A. Ahmad, An almost sure convergence theorem for weighted sum of random elements in separable Banach spaces with random weights, Sankhyā Ser. A 44 (1982), no. 2, 262-268. MR 84c:60010. Zbl 586.60008.

[2] B. D. Choi and S. H. Sung, Almost sure convergence theorems of weighted sums of random variables, Stochastic Anal. Appl. 5 (1987), no. 4, 365-377. MR 88m:60079. Zbl 633.60049.

[3] Y. S. Chow and T. L. Lai, Limiting behavior of weighted sums of independent random variables, Ann. Probab. 1 (1973), 810-824. MR 50 5909. Zbl 303.60025.

[4] W. J. Padgett and R. L. Taylor, Convergence of weighted sums of random elements in Banach spaces and Fréchet spaces, Bull. Inst. Math. Acad. Sinica 2 (1974), 389-400, Collection of articles in celebration of the sixtieth birthday of Ky Fan. MR 5011360. Zbl 301.60004.

[5] A. I. Sakhanenko, On Lévy-Kolmogorov Inequalities for Banach-space-valued Random Variables, Theory Probab. Appl. 29 (1984), 830-836.

[6] R. L. Taylor, Stochastic convergence of weighted sums of random elements in linear spaces, Lecture Notes in Mathematics, vol. 672, Springer-Verlag, Berlin, 1978. MR 80g:60006. Zbl 443.60004 .

[7] W. A. Woyczyński, On Marcinkiewicz-Zygmund laws of large numbers in Banach spaces and related rates of convergence, Probab. Math. Statist. 1 (1980), no. 2, 117-131. MR 82i:60026. Zbl 502.60006.

Hu: Department of Mathematics, National Tsing Hua University, Hsinchu, 30043, TAIWAN

Chang: InSTitute OF MATHEMATICS, ACADEMIC Sinica, TAIPEI, 11529, TAIWAN 


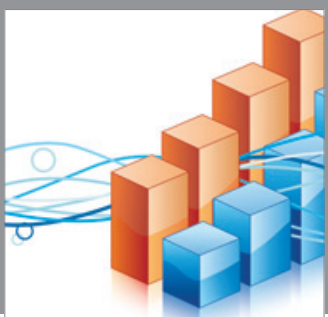

Advances in

Operations Research

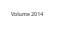

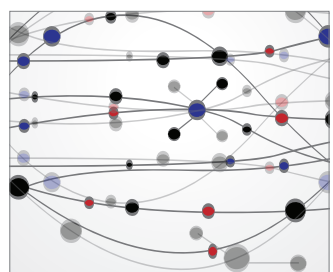

\section{The Scientific} World Journal
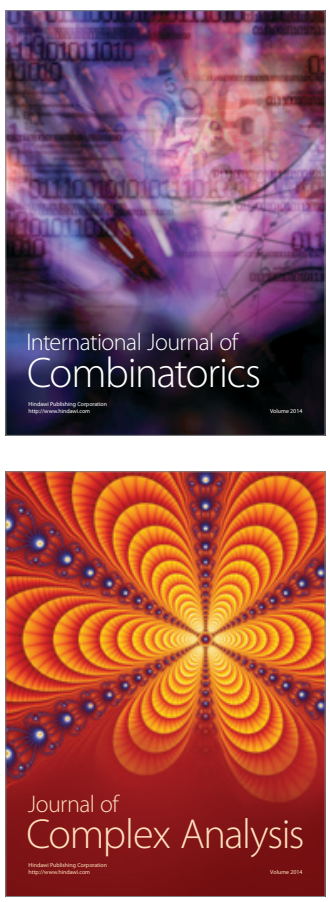

International Journal of

Mathematics and

Mathematical

Sciences
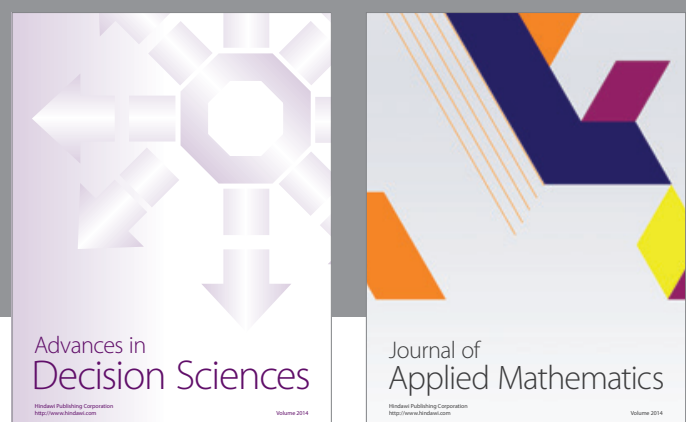

Journal of

Applied Mathematics
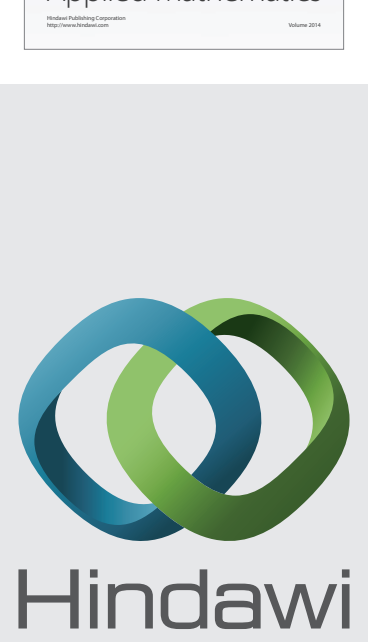

Submit your manuscripts at http://www.hindawi.com
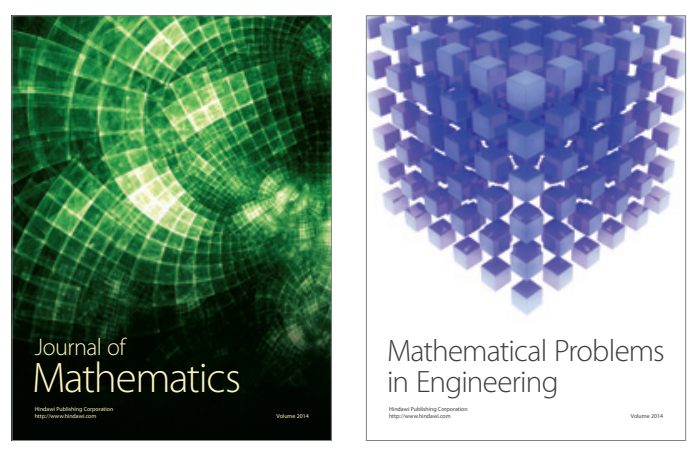

Mathematical Problems in Engineering
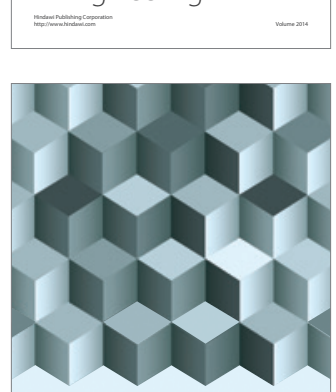

Journal of

Function Spaces
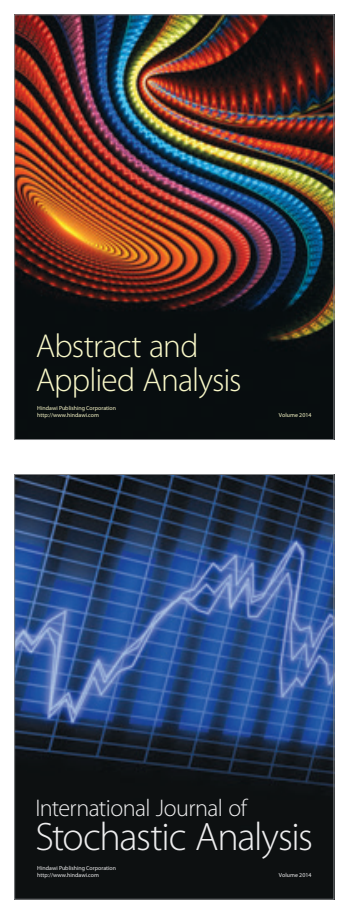

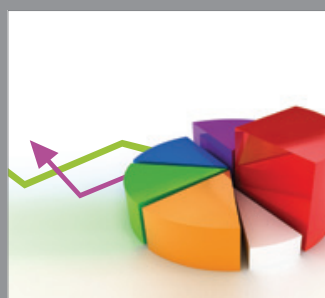

ournal of

Probability and Statistics

Promensencen
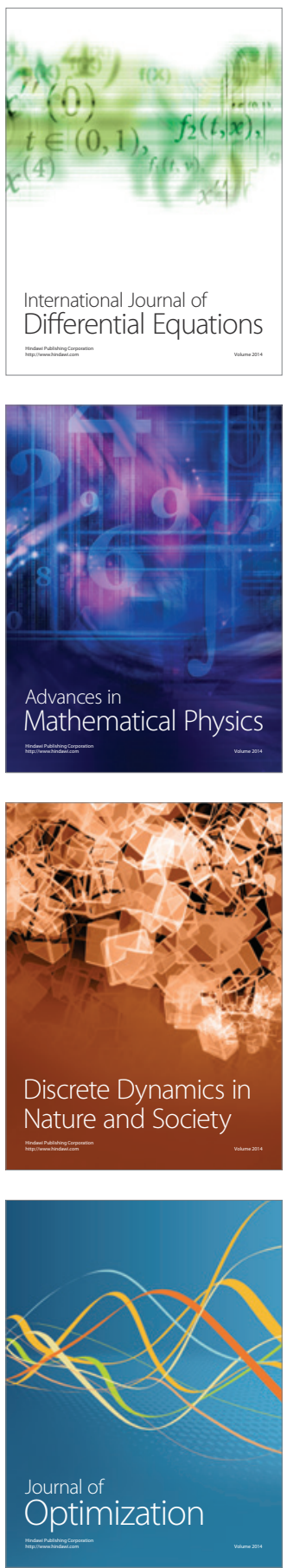